\title{
La Grande Peur et le complot aristocratique sous la Révolution française
}

\section{Timothy Tackett}

\section{Q OpenEdition \\ 1 Journals}

\section{Édition électronique}

URL : https://journals.openedition.org/ahrf/1298

DOI : 10.4000/ahrf.1298

ISSN : 1952-403X

Éditeur :

Armand Colin, Société des études robespierristes

\section{Édition imprimée}

Date de publication : 1 mars 2004

Pagination : 1-17

ISSN : 0003-4436

\section{Référence électronique}

Timothy Tackett, « La Grande Peur et le complot aristocratique sous la Révolution française », Annales historiques de la Révolution française [En ligne], 335 | janvier-mars 2004, mis en ligne le 20 février 2008, consulté le 22 avril 2022. URL : http://journals.openedition.org/ahrf/1298 ; DOI : https://doi.org/

10.4000/ahrf.1298

Ce document a été généré automatiquement le 22 avril 2022.

Tous droits réservés 


\title{
La Grande Peur et le complot aristocratique sous la Révolution française
}

\author{
Timothy Tackett
}

1 Parmi les études traitant de la période révolutionnaire, rares sont celles qui ont autant maitrisé le sujet que la célèbre monographie de Georges Lefebvre, La Grande Peur ", publiée en 1932. Reconnu dès sa parution comme un véritable classique - pour l'ampleur de sa base empirique autant que pour la subtilité et la diversité de son analyse -, l'ouvrage a marqué l'abandon quasi total des recherches sur le sujet, et ce pendant près d'un demi-siècle ${ }^{2}$. Bien sûr, Georges Lefebvre n'est pas le premier à avoir reconnu l'importance de cet extraordinaire mouvement de panique qui secoua la France à la fin de juillet et au début d'août 1789, notamment son impact sur la formation de la garde nationale et sur la renonciation au "féodalisme" par l'Assemblée nationale lors de la Nuit du 4 août. Mais pendant près d'un siècle et demi, on s'en est tenu à l'idée que l'événement s'était déclenché à peu près partout en même temps et qu'il avait été consciemment fomenté par des éléments appartenant ou bien à la noblesse ou bien aux patriotes. Ce n'est qu'après des années de recherches assidues que Lefebvre put démontrer irrévocablement que la Peur, en fait, n'était pas un phénomène simultané, mais qu'elle consistait plutôt en une série de paniques en réactions en chaîne provenant de cinq ou six sources et qui se déroulaient pendant une période de trois semaines.

2 Non content de déterminer l'origine et les modalités de la panique, Lefebvre tenta également de sonder l'état psychologique des Français et des Françaises à la veille de l'événement, expliquant ainsi leur empressement à donner crédit aux rumeurs selon lesquelles des milliers de soi-disant "brigands" allaient attaquer leurs communautés. Par une analyse aussi subtile que complexe, il étudia minutieusement la crise de subsistances, la peur grandissante envers les vagabonds et mendiants, les vagues d'émeutes locales et l'espoir suscité par la convocation des États généraux. Il découvrit que la Grande Peur avait en fait été précédée par un grand nombre de mini-paniques 
locales, provoquées par la localisation de brigands imaginaires dès le mois de mai et ce jusqu'à la veille de la panique générale de la fin juillet. Pourtant, selon ses propres dires, Lefebvre n'était pas convaincu que la somme de ces facteurs suffisait à expliquer l'ampleur des événements de fin juillet. Il pensait qu'il devait y avoir un "multiplicateur" supplémentaire, qui aurait transformé les nombreuses peurs locales en un traumatisme national. Il finit par se convaincre que l'élément critique était la soudaine crainte d'un "complot aristocratique", la conviction que des groupes de nobles embauchaient ces brigands tant redoutés pour dévaster les champs juste avant la récolte et se venger ainsi du tiers état en instaurant la famine. "Dans la seconde quinzaine de juillet [écrit Lefebvre] entre les innombrables causes d'insécurité qui alarmaient le royaume et le "complot aristocratique", la synthèse se réalise brusquement et c'est la cause déterminante de la grande peur ». Sans ces rumeurs de complot aristocratique, « la Grande Peur serait difficilement concevable ${ }^{3}$.

3 Au départ, dans son introduction, Lefebvre maintient que son interprétation n'est pas définitive et qu'elle peut être considérée comme une sorte d'hypothèse de travail : « en signalant les questions à résoudre et en suggérant des solutions, on a une chance de susciter et d'orienter des recherches nouvelles " ${ }^{4}$. Or, il poussera l'idée un peu plus loin six ans plus tard dans sa synthèse influente, Quatre-vingt-neuf. La prétendue obsession nationale pour un complot aristocratique sera alors considérée comme fondamentale à la politisation de la France rurale ainsi qu'à l'achèvement de la "quatrième étape" des origines de la Révolution, la radicalisation de la paysannerie. En juillet 1789, affirme Lefebvre, "l'idée du "complot aristocratique" naît et s'enracine avec plus de force encore [chez les paysans] que chez les bourgeois" " ${ }^{5}$. Si tous les chercheurs ne vont pas jusque là, la thèse du "complot aristocratique" a, en fait, été acceptée par bon nombre d'historiens de toutes positions idéologiques, de Georges Rudé et Albert Soboul à François Furet et Simon Schama ${ }^{6}$.

4 Dans cet article, nous voudrions réexaminer le fondement de la notion de complot aristocratique à la veille de la Grande Peur. Nos observations reposent en partie sur une série d'études ultérieures à la publication des principaux écrits de Lefebvre, et, plus précisément, sur des travaux parus depuis ces quinze dernières années. Parmi ceux-ci, les ouvrages de Clay Ramsay et de John Markoff sont particulièrement utiles ${ }^{7}$. Mais nous utilisons également une grande variété d'études locales et de documents imprimés relatant l'expérience de la Grande Peur par des particuliers ${ }^{8}$. Cette étude nous permet de conclure que les preuves soutenant la célèbre thèse de Lefebvre sont loin d'être convaincantes. Nous proposons enfin, brièvement, une autre explication de ce mouvement de panique ainsi que quelques réflexions finales sur les implications d'une telle révision pour notre compréhension du processus de la Révolution française.

5 Les principaux arguments de Lefebvre en faveur de l'idée d'un complot aristocratique proviennent de Paris. Citant des articles de journaux, la correspondance des députés et les rapports d'opinion publique d'un agent du ministre des Affaires étrangères, il apporte les preuves solides d'une "synthèse qui se réalise brusquement" dans la capitale à la veille de la crise de la mi-juillet. Le « complot de famine », pour reprendre les termes de Steven Kaplan, constitua, pendant des siècles, un thème répandu de la mentalité collective parisienne, et les pénuries de grain du printemps 1789 ne firent qu'intensifier considérablement ces anxiétés. Lorsqu'il devint manifeste que la grande majorité des députés nobles était opposée aux réformes massives qu'allait introduire le tiers état, les Parisiens commencèrent à craindre les réactions et représailles des 
aristocrates. Le rassemblement des troupes mercenaires tout autour de la capitale, début juillet, et la destitution aussi soudaine qu'inattendue de Necker alimentèrent les preuves d'un projet de conspiration destiné à mettre un terme à la Révolution. De fait, ayant débuté le 11 juillet, l'insurrection de Paris peut être en partie considérée comme une réponse de peur vis-à-vis des rumeurs relatives à l'imminence d'une émeute contre-révolutionnaire. La première vague d'émigration des nobles, qui commença immédiatement après la prise de la Bastille, ne fit qu'accentuer l'appréhension du peuple quant à l'éventualité d'une alliance entre les grands aristocrates et les puissances étrangères ${ }^{9}$. Le 21 juillet, Bailly, le maire, généralement peu réceptif aux thèses de complot, déclara qu'il soupçonnait que les prix élevés du grain fussent "l'effet d'une combinaison odieuse pour asservir de plus en plus la métropole au despotisme ministériel ${ }^{10}$. L'obsession atteignit son paroxysme vers la fin du mois avec les nouvelles de l'explosion du château de Quincey en Franche-Comté, les rumeurs selon lesquelles les Anglais s'apprêtaient à s'emparer du port de Brest, les récits de brigands localisés dans la périphérie et dans la ville même, et les échos selon lesquels d'autres brigands coupaient le blé encore vert juste au nord de Paris dans la région de Soissons. C'est précisément dans ce contexte que l'Assemblée constituante établit les Comités de recherche et des rapports, chargés en partie de démasquer les complots ${ }^{11}$. Si l'expression "complot aristocratique" ne semble guère utilisée dans la rhétorique de l'époque, il n'en reste pas moins incontestable qu'un grand nombre de Parisiens, à tous les échelons de la hiérarchie sociale, soupçonnait les ministres de l'Ancien Régime et autres vils courtisans - tous "aristocrates" - d'ourdir un complot.

6 Mais jusqu'à quel point une telle vision de la situation avait-elle pénétré la province avant l'explosion de la Grande Peur? Là, l'évidence présentée par Lefebvre est beaucoup plus mince. À la lumière de plusieurs centaines de lettres adressées à l'Assemblée nationale naissante, Lefebvre put témoigner de l'hostilité croissante que l'élite des villes de province manifestait à l'encontre de la noblesse. Toutefois, rares sont ses preuves quant à la croyance au "complot aristocratique" qui aurait déclenché la Grande Peur ${ }^{12}$. En outre, il atteste ailleurs dans son ouvrage des difficultés de communication et de la confusion avec laquelle les nouvelles se propageaient en ces débuts de Révolution. Les journaux révolutionnaires, tout juste créés, étaient encore mal distribués en province, et même les événements les plus importants pouvaient mettre plusieurs jours avant de gagner toutes les villes. Généralement, comme le remarque Lefebvre, les sources d'information les plus rapides et les plus fiables procédaient des députés, dont les lettres envoyées aux comités de correspondance locaux étaient souvent lues en public dans les villes ${ }^{13}$. Le problème est que dans la correspondance qui nous est parvenue, la plupart des députés ne font allusion à des complots qu'à la fin de juillet, alors que l'Assemblée débattait de la création des deux nouveaux comités d'investigation. Ces lettres ont dû arriver à destination beaucoup trop tard pour avoir pu exercer une influence sur la psychologie de la Peur en province ${ }^{14}$.

7 Les éléments mis en avant par Lefebvre pour démontrer que les rumeurs d'un complot aristocratique s'étaient propagées à la campagne - où résidait $80 \%$ de la population prêtent encore plus à discussion. Il ne mentionne que quelques lettres issues de certaines villes, une déclaration isolée du comte de Puységur et plusieurs notes de curés relevées dans les registres paroissiaux. On constatera du reste que ces dernières étaient en fait souvent consignées à la fin de chaque année, et peuvent aisément représenter des interprétations rétrospectives des événements passés. D’ailleurs, 
Lefebvre a lui-même reconnu l'insuffisance de sa documentation, observant qu'il n'y avait au bout du compte aucun moyen de savoir ce que les paysans pensaient ou disaient à l'époque ${ }^{15}$. Cela étant, si nous n'avons aucune preuve directe de l'état d'esprit de la paysannerie, nous possédons en revanche un grand nombre de descriptions du phénomène de panique au moment où il gagnait la province, rédigées par quelques membres de l'élite rurale et urbaine. Bien qu'il ait accumulé une documentation considérable sur la présence et la propagation de la Peur, Lefebvre n'a réalisé aucune étude systématique de ces descriptions, ni des véritables objets de la peur telle qu'elle avait été relatée par des témoins. Qui étaient ces prétendus "ennemis", soupçonnés d'attaquer les communautés provinciales, et qu'étaient les objectifs qu'on leur imputait?

8 Quand nous nous penchons sur ces récits, écrits immédiatement avant ou pendant la panique, nous ne trouvons que de très rares indications estimant que les "brigands" sont à la solde des aristocrates. Nous avons découvert quelques références de cet ordre dans les villes du sud de la Champagne - notamment à Bar-sur-Seine et à Ervy -, portant sur « la ligue formée par les aristocrates » ou « une conspiration à jamais exécrable». L'on sait, cependant, que les deux villes en question ont entretenu une correspondance directe avec les Électeurs de Paris peu de temps avant la rédaction de ces récits ${ }^{16}$. Et autant qu'on puisse en juger, les références de cette nature restent extrêmement rares, en particulier à la campagne, et quasiment inexistantes en dehors du Bassin parisien.

9 La transformation des prétendus brigands en une armée étrangère conduite par ou à la solde de princes aristocratiques est une idée plus répandue. On trouve quelques récits de cet ordre dans le sud-est de la France où d'aucuns annoncent l'invasion de troupes savoyardes menées par le comte d'Artois. Autant qu'on puisse en juger, cette nouvelle émergea dans le nord du Dauphiné et se propagea sporadiquement dans le Languedoc et la Provence. Quelques villes du Vivarais commencèrent par annoncer «la nouvelle de l'invasion inopinée de 10000 Piémontais, conduits par le comte d'Artois, qui pillent et brûlent tout sur leur passage ». Ailleurs, un propriétaire aisé de la région de Cahors note dans son journal que l'ennemi consiste en 40000 soldats menés par le comte d'Artois, espérant "dompter le tiers État ", étant donné que celui-ci veut imposer les nobles ${ }^{17}$.

10 Mais autant dans le Vivarais que dans le Dauphiné, le parallèle établi entre les troupes d'invasion étrangères et les aristocrates français semble avoir perduré surtout dans un certain nombre de villes. En dehors de ces villes, les descriptions du prétendu ennemi se rapprochent davantage du "brigand", beaucoup plus envahissant. Et dans la grande majorité des cas, en particulier hors des villes, la transformation des brigands en soldats ne relève nullement d'une quelconque autorité aristocratique. Il est fort probable que ces rumeurs ne faisaient que refléter la mémoire et les peurs collectives, particulièrement tenaces aux abords des côtes et des frontières - peur des Anglais dans l'ouest de la France, des Espagnols près des Pyrénées, des "Maures" en Provence, etc. En outre, les descriptions font preuve d'une curieuse fluidité, évoluant continuellement et se transformant au gré des différentes versions de "l'ennemi" imaginé, tandis que la panique se répand dans les campagnes. Dans le Dauphiné, la transformation des "brigands" en "Savoyards" se produit précisément au moment où la Peur traverse la ville des Abrets, à la frontière de la Savoie. Plus au sud, ainsi que dans les régions moins vulnérables et plus éloignées des frontières, les histoires d'invasion seront souvent “corrigées" pour signaler l'arrivée de brigands, ou « voleurs et bandits » ${ }^{18}$. 
11 Nous avons également trouvé des cas dans lesquels la rumeur initiale d'une armée en marche procédait d'une certaine déduction logique. Aux yeux des individus assaillis de récits relatifs à d'énormes bandes de brigands venues de plusieurs directions à la fois, il ne pouvait s'agir que d'armées organisées. Ainsi, quand un notaire de Luzech entend dire que 40000 brigands s'approchent par deux routes différentes, il en tire rapidement la conclusion qu'il ne s'agit point de brigands mais de deux forces d'invasion étroitement coordonnées. Et c'est le message qu'il transmet à ses amis et voisins. Dans tous les cas, les objets de leur terreur sont souvent doués d'une extraordinaire versatilité : le curé de Champniers, dans le Limousin, prend note des descriptions que lui ont faites ses voisins : «Les uns disent que ce sont les Anglais, d'autres que ce sont des Pandours, des échappés des galères, des voleurs, ou des brigands ${ }^{19}$.

En fait, à la lumière de notre lecture, l'écrasante majorité des observateurs confrontés à la menace imminente utilise le mot "brigands", ou un synonyme proche, sans aucune indication relative à une quelconque conspiration aristocratique. Ils qualifient leurs prétendus "ennemis" de criminels malveillants agissant pour leur propre compte et tirant profit du chaos environnant pour parvenir à leurs fins. Les autres qualificatifs sont tout aussi significatifs : le deuxième terme le plus employé pour décrire le péril est "bandits". Ils sont tour à tour des «brigands et bandits » (Aubusson), des « ravageurs, incendiaires et mutilants» (Lauzerte), des "gens sans aveu» (Touraine), des «malfaiteurs échappés des bagnes, de vrais bandits venus de tous les pays» (Basse Auvergne) ${ }^{20}$.

13 Une catégorie particulière de malfaiteurs consistait en brigands que l'on disait se déplacer de village en village pour détruire les récoltes. Ce fut la première version de la Peur que reçut l'Assemblée nationale en provenance du Soissonnais. Comme nous l'avons déjà vu, de nombreux députés et Parisiens émirent immédiatement l'hypothèse que ces brûleurs de récolte étaient à la solde de ministres ou d'aristocrates. Or, si l'on examine les récits contemporains des témoins de la Peur dans la France rurale, on ne trouve pratiquement jamais un tel rapprochement. La scrupuleuse analyse sémantique de Clay Ramsay, qui étudie l'usage des mots dans le Soissonnais pendant la panique, suggère que le terme générique de "brigands" servait à qualifier toutes sortes d'éléments indésirables, en fonction de la région et de la classe sociale concernées. Mais il ne trouve aucune référence à une quelconque connexion entre les brigands et les nobles, et conclut qu'il "parait peu probable que le "complot aristocratique" ait joué un rôle pendant la Grande Peur » dans le Soissonnais ${ }^{21}$. En fait, la crainte des brigands brûleurs de récolte semble étroitement liée à une autre anxiété, omniprésente dans maintes régions de France au cours des mois qui précédèrent la Révolution, et parfaitement décrite par Lefebvre dans son ouvrage: la peur de ces bandes de vagabonds qui avaient de plus en plus recours aux menaces et aux extorsions pour soutirer la "charité" aux habitants des campagnes ${ }^{22}$.

En outre, d'après les études et les documents imprimés locaux, les rumeurs selon lesquelles les brigands détruisaient les récoltes restaient essentiellement confinées dans la moitié nord du royaume, comme en témoignent les innombrables récits de saccage de récoltes en Artois, Picardie, Champagne, Île-de-France et dans le Maine ${ }^{23}$. Au sud de la Loire, en revanche - dans le Poitou, le Limousin, l'Auvergne, le Dauphiné, la Provence ou l'Aquitaine, entre autres -, nous ne trouvons pratiquement aucune référence de cet ordre ${ }^{24}$. Les raisons de cet étrange contraste restent incertaines et méritent un examen plus approfondi. Peut-être cette absence de peur provient-elle du 
fait que les céréales parvenaient plus tôt à maturité dans le Midi et avaient été récoltées avant que le mouvement de panique n'apparaisse. De surcroît, les vagues de panique tendaient à arriver dans le Sud quelques jours plus tard que dans le Nord. Enfin, on peut imaginer que dans les zones de polyculture, très répandues dans les régions méridionales, les menaces exercées sur les récoltes de céréales provoquaient moins d'anxiété que dans le Nord, où la monoculture prévalait. Quoi qu'il en soit, dans l'imaginaire du sud de la France, les "brigands" de juillet et d'août canalisaient leur rage presque exclusivement sur les bâtiments et les personnes plutôt que sur les récoltes.

Bien sûr, il convient de distinguer les descriptions de "l'ennemi" rédigées au moment de la panique des réflexions faites après coup. Lorsqu'il devint manifeste qu'aucun brigand ne s'était matérialisé - ou, comme d'aucuns l'affirmèrent, qu'ils «s'étaient à présent retirés » - on entreprit de comprendre et d'expliquer ce qui s'était passé. Et ce, plus particulièrement, lorsqu'on s'aperçut, par les récits de voyageurs et les lettres d'amis, que des paniques similaires avaient secoué bien d'autres régions du pays. Dans le département de l'Ain, par exemple, constatant qu'aucun brigand ne s'était montré, chacun commença à se demander ce qui s'était passé, et conclut qu'il avait été « le jouet d'une mauvaise plaisanterie ${ }^{25}$. Mais qui était donc à l'origine de cette révoltante et coûteuse rumeur?

C'est à ce moment précis, après l'événement, qu'un certain nombre d'individus combien exactement, on ne saurait le dire - en vint à soupçonner la noblesse d'avoir déclenché la panique. Un juge de Brive décrit ainsi les conjectures dont la Grande Peur fit l'objet dans sa ville : les uns attribuent la peur « aux menées du corps de la noblesse fort mécontente de la tenue des États généraux et des résolutions prises contre elle par les communes; les autres aux Anglais répandus en France; et la plupart à quelques seigneurs de la cour, aussi mécontents du tiers état » (le magistrat lui-même en vint à la conclusion que Dieu seul pouvait avoir provoqué une telle panique, pour des motifs qu'il ne parvenait pas à deviner) ${ }^{26}$. Dans certaines parties du royaume, cette réflexion post mortem fut probablement à la fois la cause et la conséquence de la méfiance grandissante qu'entretenaient les habitants des petites villes et les paysans à l'encontre de la noblesse. Ainsi, dans certaines régions, ce sont les séquelles de la Grande Peur plutôt que la crainte antérieure d'un complot aristocratique - qui marquèrent une étape décisive dans l'évolution des attitudes envers la noblesse au cours de l'année 1789.

Parfois, la simple suspicion faisait place à une véritable violence anti-aristocratique. Les cas les plus dramatiques, sans aucun doute, eurent lieu dans le Viennois et le Vivarais. Quand ils apprirent qu'il n'y avait ni brigands ni armée savoyarde, les paysans de certains villages du Viennois conclurent - là encore après les événements - que c'étaient les seigneurs qui avaient dû répandre de telles rumeurs. Et pour se venger de cette "trahison", des bandes entières commencèrent à attaquer, et parfois à brûler, les châteaux environnants. Il se produisit plus ou moins la même chose dans certaines parties du Vivarais, peut-être sous l'influence des événements du Dauphiné : « Furieux d'avoir tant couru pour rien [décrit Jolivet] leur déception se transforme vite en colère ». Bientôt, des rumeurs relatives à "la connivence" entre les nobles et ces brigands qui n'avaient jamais paru commencèrent à circuler ${ }^{27}$.

Mais dans le cadre plus large du royaume tout entier, les cas rencontrés dans le Viennois et le Vivarais font figure d'exception. Ailleurs, chaque fois que la panique 
engendrait une violence anti-aristocratique, celle-ci se limitait presque toujours à des attaques contre des individus particuliers. En Champagne, dans le Poitou, en Auvergne, dans la région de Toulouse et dans le Sud-Ouest en général - régions pour lesquelles nous disposons d'études approfondies sur la Peur et ses conséquences - les historiens n'ont trouvé que quelques cas d'attaques anti-aristocratiques qui soient liées à la Grande Peur ${ }^{28}$. S'il faut encore poursuivre des recherches avant de tirer des conclusions définitives, il semble toutefois que la plupart des agressions provoquées par ce type d'incidents visaient les seigneurs locaux - haïs depuis longtemps pour toutes sortes de raisons - plutôt que la noblesse ou le système féodal en général.

De fait, comme l'ont remarqué plusieurs historiens, la Grande Peur a souvent été tout aussi révélatrice de la solidarité verticale qui unissait les groupes sociaux, qu'ils soient roturiers ou privilégiés, que de l'animosité anti-aristocratique ${ }^{29}$. À maintes occasions, cette solidarité semble même avoir été renforcée par l'expérience de la panique. Peutêtre sera-t-il un jour possible de dresser le bilan géographique des chefs des milices locales créées dans tout le pays pour répondre à l'urgence posée par l'approche des brigands. Mais il existe, très clairement, de nombreux exemples de paysans ou de citadins ayant recruté des nobles afin que ceux-ci dirigent la défense de leurs communautés. Ceci se vérifie tout particulièrement dans les villages, quoi que dans bon nombre de villes de petite ou moyenne taille - comme Montoire, Mamers, Brive, Limoges, Cahors, etc. -, les milices fussent placées sous l'autorité d'anciens officiers nobles. Parfois même, la quasi intégralité de l'autorité était confiée à l'aristocratie locale ${ }^{30}$. En Artois, l'expérience de la Peur «ne cause aucune émeute, loin de là, elle scelle au contraire, entre tous les citoyens, l'union la plus étroite pour la défense de leur vie et la protection de leurs biens menacés ». De semblables alliances entre nobles et non nobles ont été identifiées dans des régions aussi distinctes que le Soissonnais, le Maine, le Gévaudan et l'Aquitaine ${ }^{31}$.

20 Bien sûr, s'il est si difficile d'interpréter l'agitation populaire qui marqua le mois de juillet 1789, c'est, en partie, parce qu'il est très malaisé de démêler les cas de panique collective des épisodes tout aussi perturbateurs d'insurrections rurales qui se produisirent juste avant ou après la Grande Peur. Localisées dans des régions très diverses, les révoltes paysannes de ce mois touchèrent une superficie globale de loin inférieure à celle affectée par la Grande Peur. Aux rébellions ci-dessus mentionnées du Viennois et du Vivarais, nous devons ajouter cinq autres révoltes, qui se déclarèrent seulement une ou deux semaines auparavant: celles de la Franche-Comté, de l'Alsace, du Mâconnais, de la Basse-Normandie et du Hainault ${ }^{32}$. Les recherches menées entre autres par Anatoli Ado, John Markoff et Jean Nicolas, ont bien renouvelé nos connaissances du contexte des actions collectives populaires au débuts de la Révolution. De toute évidence, les émeutes, urbaines et rurales, perdurèrent pendant tout l'hiver et le printemps 1788-1789. Bien qu'un certain nombre d'entre elles aient été dirigées contre les nobles, les nouvelles études laissent à penser que la préoccupation majeure des insurgés était liée à la pénurie de nourriture. Ainsi, pour Markoff, elles «constituaient probablement la plus grande vague d'émeutes alimentaires qu'ait jamais connu la France jusqu'à cette époque ${ }^{33}$.

21 Le mois de juillet, cependant, vit une hausse considérable des émeutes antiaristocratiques ${ }^{34}$. Chacun, parmi les sept soulèvements principaux, possédait sa propre dynamique, son lot particulier de causes et de motifs ${ }^{35}$. Dans certains cas, les gens dirigeaient leur haine non plus contre des nobles en particulier, mais contre tout le 
système seigneurial. Ce fut manifestement le cas de la Franche-Comté, par exemple, où la révolte anti-aristocratique couvait depuis avril. Dans le Vivarais et en BasseNormandie, en revanche, les attaques semblaient viser tous les groupes et individus que l'on soupçonnait d'amasser le grain ou d'exploiter plus généralement les classes inférieures: seigneurs, administrateurs royaux, percepteurs, membres du clergé, bourgeois ayant récemment gagné des procès et fermiers exploitant des terres que les paysans considéraient comme communales ${ }^{36}$. En Basse-Normandie, la crise économique et de subsistances locale semble s'être considérablement intensifiée par la réduction des dépenses allouées par le gouvernement pour la construction du port de Cherbourg. En Franche-Comté, les insurrections étaient en partie influencées par les âpres luttes intestines qui opposaient différentes factions de nobles, chacune dénonçant publiquement les forfaits de l'autre. Il n'est d'ailleurs pas impossible qu'une intense politisation antérieure ait également affecté le Mâconnais et le Dauphiné à la veille des révoltes.

Dans certains cas, il put y avoir des liens entre les rébellions et la Grande Peur proprement dite. Les courants de panique qui secouèrent la Normandie et la FrancheComté étaient probablement étayés par des épisodes de violence locale : les bandes de paysans en colère qui attaquaient les châteaux ou cherchaient de la nourriture, en effet, se voyaient transformées en brigands en marche. Dans le Viennois et le Vivarais, comme nous l'avons déjà vu, la corrélation se faisait dans l'autre sens, l'arrivée de la Peur déclenchant des assauts contre les seigneurs locaux. Dans le Mâconnais, où des vagues de la Grande Peur semblent avoir déferlé au moment même où éclataient les révoltes de paysans et de viticulteurs, le rapport était plus complexe et fonctionnait peut-être, dans une certaine mesure, dans les deux sens. Mais en général, et c'est l'un des thèmes de l'ouvrage de Lefebvre, les zones de rébellion étaient isolées et indépendantes des vagues de panique environnantes ${ }^{37}$. Ainsi, alors que la Peur sévissait avec intensité à Mâcon même et dans les régions situées à l'est de la Saône, elle semble avoir à peine pénétré les secteurs touchés par les révoltes. Seuls deux villages de ce secteur furent mobilisés par la Peur, et, dans les deux cas, l'agitation fut presque immédiatement redirigée - par l'influence qu'exerçaient les villages voisins - vers une attaque contre l'aristocratie locale. L'existence parallèle et séparée des deux phénomènes semble se vérifier également, à très petite échelle, dans le Soissonnais de Ramsay, où les zones d'émeutes semblent avoir été épargnées et ignorantes des vagues de peur qui déferlaient tout autour d'elles ${ }^{38}$.

Expliquer l'incompatibilité de ces deux types de mouvements collectifs ruraux paniques et insurrections -, tels qu'ils se déroulèrent en juillet 1789, nécessiterait une analyse approfondie, analyse que Lefebvre lui-même n'entreprit jamais. Mais il est clair que ces mouvements reflètent deux dynamiques sociales très distinctes ainsi que deux paradigmes d'interprétation et modes d'investissement émotionnel différents. Les paniques étaient en grande partie gouvernées par la peur d'ennemis externes et essentiellement inconnus; les insurrections reposaient probablement plus sur la colère que sur la peur et étaient poussées par la conviction que des individus internes à la communauté étaient à l'origine de leurs souffrances. Quand de telles révoltes se produisaient, elles semblent avoir attiré tellement l'attention sur les ennemis internes que d'importantes parties de la population devinrent imperméables à la possibilité de menaces extérieures ${ }^{39}$. 

nombreux historiens - amalgamèrent les deux différents types de mouvements pour en faire un soulèvement général contre le système seigneurial. Les Constituants étaient invariablement impressionnés par les descriptions de châteaux en feu et de violence menée contre les seigneurs - notamment après que quelques députés apprirent que leurs propres résidences avaient été la proie des flammes -, quand bien même ces attaques affectaient des zones probablement dix fois moins importantes que celles touchées par la Grande Peur. Cette vision d'une conflagration générale apparaissant presque simultanément dans tous les recoins du royaume joua un rôle déterminant dans le déclenchement de la Nuit du 4 août. Paradoxalement, le principe de la "suppression du féodalisme" prôné par l'Assemblée - qui maintenait toutefois les droits féodaux jusqu'à ce que la "propriété" en question ait été remboursée par les paysans favorisa certainement l'expansion et l'intensité de l'animosité anti-aristocratique, même dans les régions où une telle animosité ne s'était encore jamais ouvertement manifestée ${ }^{40}$.

Tout ceci nous éloigne quelque peu de notre sujet, et une question aussi évidente que difficile demeure: si ce n'est la suspicion d'un complot aristocratique qui peut expliquer la Grande Peur, alors qu'est-ce donc? Quel est le catalyseur - ou le «multiplicateur ", pour reprendre le terme de Lefebvre - qui canalisa les innombrables et obscurs éléments d'anxiété, ainsi que les nombreuses mini-paniques locales du printemps 1789, en une extraordinaire terreur en chaîne, dans le royaume tout entier, pendant la seconde quinzaine de juillet? Nous soutenons pour notre part - sous forme d'hypothèse de travail - que, pour la grande majorité des gens, le déclencheur ne fut pas la brusque synthèse d'un complot aristocratique, mais la terreur d'une anarchie imminente, de l'effondrement de toutes les forces de l'ordre public - autant à Paris qu'en province - qui découla directement de la crise de la mi-juillet.

S'il nous reste peu de temps pour développer cette hypothèse, il convient toutefois d'insister sur l'expérience émotionnelle complexe que vécurent les citoyens français pendant cet extraordinaire printemps et été 1789. Les historiens ont fait valoir à juste titre l'optimisme et l'exaltation des débuts de la Révolution. Il ne fait aucun doute qu'un grand nombre de gens - issus en particulier des classes moyennes urbaines, mais aussi de certaines catégories de paysans - furent exaltés, électrisés par les développements aussi spectaculaires qu'inattendus de 1789 : la convocation des États généraux par le roi et la "régénération de la nation française " qui s'ensuivit, phénomène sans précédent dans l'histoire du royaume. Or, cet enthousiasme quasi millénariste cachait presque toujours des sentiments d'incertitude et de doute profond devant les ramifications politiques et sociales du programme des réformes à venir et les transformations phénoménales que ces réformes allaient impliquer. Cette confusion de sentiments était particulièrement manifeste chez les députés à Versailles, lesquels faisaient souvent part de leurs incertitudes émotionnelles - «les contrastes saisissants entre le bien et le mal, l'espoir et la peur, la joie et la tristesse » - dont beaucoup, ainsi qu'Antoine Durand l'écrira plus tard, étaient liées à « l'anxiété de la liberté naissante » ${ }^{41}$.

27 Au début de l'été, la majeure partie de la population provinciale avait traversé une période particulièrement instable, marquée par des vagues de révoltes de subsistances et la crainte des vagabonds ${ }^{42}$. Quand les nouvelles de la crise de la mi-juillet commencèrent à s'échapper des mailles peu fiables du système de communication de

Annales historiques de la Révolution française, 335 | janvier-mars 2004 
l'époque, les vagues sentiments d'insécurité se transformèrent en la certitude d'un effondrement très réel et imminent de l'autorité. À court terme, les soulèvements et les révoltes de Paris, ainsi que la prise de la Bastille, avaient déconcerté une grande partie de la population provinciale. Il était souvent difficile de comprendre la signification et les objectifs de la violence dans la capitale - violence qui continua bien après le 14 juillet. Si la grande majorité de la population soutenait probablement la nouvelle Assemblée nationale, elle défendait également le roi, encore considéré comme le " père de la nation ». Et l'apparente capitulation de la monarchie devant le peuple parisien peuple dont la composition et les motifs, au début, restaient très flous - la laissa profondément indécise quant à l'avenir. Les peurs d'une dérive vers l'anarchie à Paris ne furent qu'exacerbées par les révolutions municipales provinciales et par l'effondrement général de la bureaucratie royale. Nombre d'intendants, subdélégués, percepteurs et magistrats du roi, terrifiés par le sort des administrateurs royaux de Paris, se cachèrent ou abandonnèrent tout bonnement leur poste, laissant un profond vide du pouvoir dans le pays ${ }^{43}$.

C'est cet effondrement de l'autorité, à la fois dans la capitale et en province, cet apparent relâchement des forces de l'ordre, conjugué au sort incertain du monarque, qui semblaient livrer les campagnes aux attaques si redoutées des "brigands". Un notable de Bourg-en-Bresse raconte l'anxiété des citadins à la réception, le 16 juillet, du premier rapport, quelque peu confus, des récents événements de Paris: «l'on était menacé d'une incursion de brigands et de gens mal intentionnés qui n'attendaient que ce moment de désordre pour se livrer au pillage ». Remarquablement similaire est la description, un jour plus tôt, des notables de Laval: "Il est à craindre que le relâchement absolu des ressorts du gouvernement n'ouvre la porte aux désordres de l'anarchie la plus monstrueuse et n'expose la vie et les biens des citoyens aux entreprises de cette foule de malintentionnés ou de brigands qui infectent depuis quelques mois les environs de la ville ${ }^{44}$. De fait, avant que ne sévisse la Grande Peur, de vagues rumeurs s'étaient déjà fait entendre dans tout le pays que des brigands étaient partis ou avaient été chassés de la capitale à la suite des soulèvements parisiens, et qu'ils se dirigeaient vers la province. Si l'on en croit des récits contemporains, ce type de rumeurs semble avoir été beaucoup plus courant en province, à la veille de la panique, que les bruits relatifs à un complot aristocratique ${ }^{45}$.

Notre intention, dans cet article, n'est nullement de dénier l'existence de sentiments anti-aristocratiques dans la France de 1789. Nous avons par ailleurs démontré que la colère dirigée contre la noblesse constituait l'un des principaux dénominateurs communs dans la rhétorique utilisée par les futurs députés du tiers état, dans les mois qui avaient précédé les États généraux. Sarah Maza a également tiré la conclusion que chez les intellectuels des villes, bien avant la Révolution, le « langage de classe le plus évident était anti-aristocratique " ${ }^{46}$. De tels sentiments avaient certainement été exacerbés dans bon nombre de villes du royaume, au cours de la mobilisation politique de 1788 et 1789. Dans les zones rurales, également, les structures de la société agraire et de l'économie créaient invariablement un potentiel d'animosité envers les seigneurs, particulièrement - mais pas exclusivement - quand les droits seigneuriaux incluaient le champart ou autres prélèvements en nature. Et pourtant, il y avait une différence de taille entre la colère ressentie contre des seigneurs en particulier et le désir de supprimer le système seigneurial tout entier. 
30 En fait, notre étude sur la dynamique de la Grande Peur suggère qu'au début de la Révolution, les sentiments ouvertement anti-seigneuriaux étaient encore inégalement répartis. Les insurrections visant la noblesse étaient relativement peu importantes avant juillet 1789 , comparées au nombre considérable des révoltes de subsistances. Même la vague des émeutes résolument anti-seigneuriales du mois de juillet ne touchèrent qu'une petite partie $d u$ royaume. Quant au phénomène relativement différent de la Grande Peur, la thèse de Georges Lefebvre prônant l'obsession générale d'un complot aristocratique ne semble pas se vérifier en dehors de Paris et d'un certain nombre de grandes villes. Nous avons découvert en effet que, dans de vastes portions $\mathrm{du}$ pays, les liens verticaux qui unissaient les paysans et les seigneurs étaient restés remarquablement forts pendant la crise. Ce qui permet de comprendre la panique de juillet est un autre type de peur : celle de l'effondrement imminent de l'ordre public et de la propagation d'une anarchie qui semblait rendre inévitable l'attaque anticipée de brigands réels ou imaginaires.

31 Une grande partie de l'historiographie de la Révolution française du $\mathrm{XX}^{\mathrm{e}}$ siècle, de Jean Jaurès et Georges Lefebvre à Albert Soboul et François Furet, a mis l'accent sur la rupture radicale de 1789. Et nul ne saurait contester le remarquable succès des célèbres décrets pris par les États généraux et l'Assemblée nationale à la fin du printemps et pendant l'été de cette même année. Mais notre brève exploration de la dynamique de la Grande Peur souligne l'importance $\mathrm{du}$ processus continu des changements révolutionnaires après 1789 , de "l'école de la Révolution ", ainsi qu'un des Constituants l'appelait ${ }^{47}$. Pour une grande partie de la population française, notamment chez les habitants des zones rurales, la transformation des attitudes vis-à-vis de la noblesse put initialement se manifester après les débuts de la révolution politique. Elle se produisit non comme un abrupt "changement de paradigme", mais évolua sur plusieurs mois, voire plusieurs années, alimentée entre autre par l'action des législateurs révolutionnaires eux-mêmes. Il ne fait aucun doute que les décrets qui suivirent la Nuit $\mathrm{du} 4$ août procédèrent en partie du traumatisme de la Grande Peur et des insurrections paysannes du mois de juillet. Mais ces décrets, en fait, aidèrent à concentrer plus que jamais l'animosité anti-aristocratique. La méfiance envers la noblesse et les craintes d'un complot aristocratique, en effet, furent probablement bien plus importantes dans les années qui suivirent 1789 qu'elles ne l'avaient été à l'époque même de la Grande Peur.

\section{NOTES}

1.Georges LefebVRe, La Grande Peur de 1789, Paris, 1932.

2.La principale exception est l'étude d'un des élèves de Lefebvre, Louis JACOB, « La Grande Peur en Artois ", A.H.R.F., 1936, pp. 123-148. Lefebvre continua à publier occasionnellement dans les A.H.R.F. des documents relatifs à la Grande Peur.

3.Georges LEFEBVRE, La Grande Peur, op.cit., pp. 58-60, 65, 75, 144.

4.Id., pp. 1-2. Voir aussi les observations de Louis JACOB, art. cit., p. 123. 
5.Georges LEFEBVRE, Quatre-vingt-neuf, nouvelle édition Paris, 1970, p. 163.

6.Georges RUDÉ, « Introduction » à Georges LEFEBVRE, The Great Fear: Rural Panic in Revolutionary France, traduit par JOAN WHITE, New York, 1973, pp. X-XI ; Albert SOBOUL, La Révolution française, Paris, 1983, p. 223 ; Simon schamA, Citizens, New York, 1989, p. 429 ; François FURET et Denis RICHET, La Révolution française, Paris, 1973, p. 86. Voir aussi Jacques REVEL, « La Grande Peur », dans Dictionnaire critique de la Révolution française, éd. François FURET et Mona OzOUF, Paris, 1988, pp. 105-112.

7.Clay RAMSAY, The Ideology of the Great Fear: The Soissonnais in 1789, Baltimore, 1992 ; John MARKOFF, The Abolition of Feudalism. Peasants, Lords, and Legislators in the French Revolution, University Park, Pennsylvania, 1996. Voir aussi Henri DINET [erronément typographié Diné], La Grande peur dans la généralité de Poitiers, Paris, 1951 ; «Les Peurs du Beauvaisis et du Valois, juillet 1789 », Paris et Ile-de-France. Mémoires, 23-24, 1972-1973, pp. 199-286 ; «Craintes, brigandages et paniques inédits des années 1789-1791 », A.H.R.F., 81, 1981, pp. 304-306 ; et «L'année 1789 en Champagne », A.H.R.F., 55, 1983, pp. 570-595. Ont également été utiles Anatoli ADO, Paysans en révolution. Terre, pouvoir et jacquerie, 1789-1794, Paris, 1996 ; et Jean NICOLAS, La Rébellion française : mouvements populaires et conscience sociale, Paris, 2002.

8.Les ouvrages suivants ont été particulièrement importants : Francisque MÈGE, « La Grande Peur [en Auvergne] », Bulletin historique et scientifique de l'Auvergne, 2e sér., 1900, pp. 140-71, 175-240 ; B. PAUMÈs, « La Grande Peur en Quercy et en Rouergue. Notes et documents ", Bulletin de la Société des études littéraires, scientifiques et artistiques du Lot, 37, 1912, pp. 29-44, 103-117, 181-200, 229-245 ; A. LECLERC, « La Grande peur en Limousin », Bulletin de la Société archéologique et historique du Limousin, 51, 1902, pp. 17-62 ; Pierre CONARD, La Grande Peur en Dauphiné (juillet-août 1789), Paris, 1904 ; M. A. PICKFORD, « The Panic of 1789 in Touraine ", English Historical Review, 26, 1911, pp. 703-723; et « The Panic of 1789 in Lower Dauphiné and Provence ", English Historical Review, 29, 1914, pp. 276-301 ; Émile CHAUDRON, La Grande Peur en Champagne méridionale, Paris, 1924 ; et Louis $\mathrm{JACOB}$, art. cit.

9.Voir Georges LEFEBVRE, La Grande Peur, op. cit., pp. 69-78. Egalement Steven L. KAPLAN, Le Complot de famine : histoire d'une rumeur au XVIIIe siècle, Paris, 1982 ; et Pierre CARON, « La Tentative de contre-révolution de juin-juillet 1789 ", Revue d'histoire moderne, 7 , 1906-1907, pp. 5-34, 649-678.

10.Jean-Sylvain BAILLY, Mémoires d'un témoin de la Révolution, éd. Berville et Barrière, 3 vol., Paris, 1821-1822, vol. 2, p. 96. Lefebvre ne cite pas Bailly.

11.Voir par exemple BAILLY, op. cit., vol. 2, pp. 159-60 ; Pierre-François LEPOUTRE, Députépaysan et fermière de Flandre en 1789. La correspondance des Lepoutre, éd. Jean-Pierre JESSENNE et Edna Hindie LEMAY, Villeneuve d'Ascq, 1998, pp. 69-71 ; François-René-Pierre MÉNARD DE LA GROYE, Correspondance (1789-1791), éd. Florence MIROUSSE, Le Mans, 1989, p.

73 ; Jacques BRISSOT, Le Patriote français, $\mathrm{n}^{\circ} 1-3$.

12.Georges LeFEBVRE, La Grande Peur, op. cit., pp. 92-106.

13.Id., pp. 79-85.

14.Par exemple, Gantheret fait mention de complots pour la première fois dans sa lettre du 26 juillet ; Bouchette, le 27 juillet ; Maupetit et Ménard le 29 juillet ; Lepoutre le 30 juillet : Ménard, p. 73 ; Lepoutre, pp. 69-71; Claude Gantheret, coll. privée de Françoise Misserey, Dijon ; François-Joseph BOUCHETTE, Lettres de François-Joseph Bouchette (1735-1810), éd. Camille LOOTEN, Lille, 1909, pp. 236-237 ; Michel-René MAUPETIT, « Lettres de Michel-René Maupetit, député à l'Assemblée nationale constituante, 
1789-91 », éd. QUERUAU-LAMERIE, Bulletin de la Commission historique et archéologique de la Mayenne, 2e sér., 19, 1903, pp. 208-210.

15.Georges LEFEVBRE, La Grande Peur, op. cit., pp. 107-117, 189. Dans Quatre-vingt-neuf, op. cit., p. 164, Lefebvre introduit un nouvel élément d'explication pour la propagation de l'idée du complot aristocratique : « le marché [...] a été le principal foyer de contamination ». Le paysan s'y rendait au moins une fois par semaine, et c'est là qu'il « a connu les nouvelles, qu'il est entré en communion d'idées avec le peuple des villes [...] ». Mais là encore, il semble peu probable que la « synthèse qui se réalise brusquement » - à Paris - ait pu se propager aussi rapidement par ce moyen. Voir Clay RAMSAY, op. cit., pp. XXIII-XXIV.

16.É. CHAUDRON, op. cit., pp. 82-83.

17.Pierre CONARD, op. cit., pp. 49-50 ; Charles JOLIVET, La Révolution dans l'Ardèche (1788-1795), L'Argentière, 1930, p. 126 ; PAUMÈs, op. cit., pp. 35-36. Le propriétaire en question se dit « laboureur ». Mais dans son journal, il compare « l'invasion » en cours à celle de Vandels en l'an 406, une référence qui n'évoque guère la culture d'un paysan. 18.CONARD, op. cit., pp. 49-50 ; JOLIVET, op. cit., p. 131 ; PICKFORD, « The Panic in Dauphiné and Provence ", art. cit., pp. 278, 285.

19.PAUMÈs, op. cit., pp. 107-109 ; LECLER, op. cit., p. 39.

20.R. H. BAUTIER, « Les Débuts de la Révolution à Aubusson », Mémoires de la Société des sciences naturelles et archéologiques de la Creuse, 29, 1944-1946, pp. 418-20 ; PAUMÈs, op. cit., p.194 ; PICKFORD, op. cit., « Touraine », p. 708 ; MÈGE, op. cit., p. 151. Voir aussi Marcel BRUNEAU, Les Débuts de la Révolution dans les départements du Cher et de l'Indre, Paris, 1902, pp. 61-62.

21.Clay RAMSAY, op. cit., pp. 138-147, 153-154. JACOB en tira plus ou moins la même conclusion pour l'Artois, une autre région où les récoltes étaient prétendument ravagées par les brigands (JАCOB, op. cit., p. 141).

22.Georges LEFEBVRE, La Grande Peur, op. cit., pp. 15-26.

23.RAMSAY, op. cit., p. 142 ; CHAUDRON, passim ; R. TRIGER, L'Année 1789 au Mans et dans le Haut-Maine, Mamers, 1889, p. 233 ; JАСOB, p. 141 ; A.H.R.F. 10, 1933, pp. 167-175.

24.L'unique référence de ce type que j'ai trouvée à ce jour au sud de la Loire est celle de Cordes-Tolosannes (Tarn-et-Garonne), où l'on mentionne des brigands brûlant "deux gerbières” : A.H.R.F. 1961, p. 98. On remarquera toutefois qu'il s'agit de grain déjà récolté, et non de blé poussant dans un champ.

25.Eugène DuBoIs, Histoire de la Révolution dans l'Ain, tome I, La Constituante (1789-1791), Bourg-en-Bresse, 1931, p. 76.

26.LECLER, op. cit., pp. 48-50.

27.CONARD, op. cit., p. 59, 63-65 ; JOLIVET, op. cit., p. 134.

28.CHAUDRON, op. cit., p. 35-36 ; Alan FORREST, The Revolution in Provincial France: Aquitaine, 1789-1799, Oxford, 1996, p. 131 ; Jacques GODECHOT, La Révolution française dans le MidiToulousain, Toulouse, 1986, pp. 82-84 ; Marquis Marie de Roux, La Révolution à Poitiers et dans la Vienne, Paris, 1910, pp. 203-204 ; Mège, p. 159.

29.Par exemple, Donald SUTHERLAND, The French Revolution and Empire. The Quest for a Civic Order, Oxford, 2003, p. 68.

30.A.H.R.F., 1964, op. cit., pp. 498-500 ; TRIGER, op. cit., p. 234 ; LECLER, op. cit., pp. 30, 48-50 ; PAUMÈs, op. cit., pp. 37, 186-187. 
31.JACOB, op. cit., p. 142. Egalement RAMSAY, op. cit., p. XXIII-XXIV, 106 ; TRIGER, op. cit., p. 234 ; Pierre-Jean-Baptiste DELON, La Révolution en Lozère, Mende, 1922, p. 33 ; FORREST, p. 129.

32.LEFEBVRE, La Grande Peur, pp. 118-44 ; ADO, pp. 126-36. Lefebvre ne compte pas le Vivarais, mentionné pourtant par Ado.

33.MARKOFF, op. cit., pp. 242, 243-49. Voir ADO, op. cit., pp. 101-102.

34.Voir tableau de MARKOFF, op. cit., p. 276.

35.Les deux paragraphes suivants s'appuient sur Félix MOURLOT, La Fin de l'Ancien Régime et les débuts de la Révolution dans la généralité de Caen, Paris, 1913, pp. 305-320 ; Fernand EVRARD, « Les Paysans du Mâconnais et les brigandages de juillet 1789 », Annales de Bourgogne, 19, 1947, pp. 108-109 ; CONARD, op. cit., chap. II et III ; Jean GIRARDOT, Le Département de la Haute-Saône pendant la Révolution. Vol. 1, Vesoul, 1973, pp. 130-44 ; Georges LEFEBVRE, Les Paysans du Nord pendant la Révolution française, Paris, 1924, pp. 356-363 ; JOLIVET, p. 135-43 ; ainsi que LEFEBVRE, La Grande Peur, pp. 118-144 et ADO, en particulier pp. 126-136.

36.LEFEBVRE lui-même révéla une complexité analogue dans le Cambrésis dans son étude antérieure sur Les Paysans du Nord (...), op. cit., pp. 356-363.

37.Par exemple, LEFEBVRE, La Grande Peur, op. cit., p. 167.

38.EVRARD, op. cit., pp. 108-110 ; RAMSAY, op. cit., pp. 107-109, 190.

39.L'hypothèse selon laquelle les paniques et les soulèvements sont gouvernés par deux processus socio-psychologiques différents est implicite dans l'analyse de Neil J. SMELSER, Theory of Collective Behavior, réimp., Londres, 1998, chap. VI ("The Panic"). Voir aussi RAMSAY, pp. 204-205 et 242-243 ; ainsi que l'article de l'auteur « Collective Panics in the Early French Revolution, 1789-1791: A Comparative perspective », French History, vol. $17, \mathrm{n}^{\circ} 2$ (2003), pp. 149-171.

40.Ce fut notamment le cas dans certaines parties du Sud-Ouest : voir Jean BOUTIER, Campagnes en émoi. Révoltes et Révolution en Bas-Limousin, 1789-1800, Treignac, 1987. Voir aussi MARKOFF, op. cit., en particulier chap. 8.

41.Antoine DURAND à Delcamp-Boytré dans Gourdan : A.E. Cahors, carton 5-56, “14 juillet" ; et la lettre à son cousin du 23 mai 1790 : A.M. Cahors, carton non classé de lettres des députés révolutionnaires, déposé à la B.M. Cahors. Voir aussi Par la volonté du peuple; comment les députés de 1789 sont devenus révolutionnaires, Paris, 1997, pp. 144-145, de l'auteur du présent article.

42.De nombreux curés ruraux, résumant l'année 1789 dans leurs registres paroissiaux, mirent l'accent non seulement sur les transformations politiques mais aussi sur la souffrance et la mortalité dues à la crise de subsistance, ainsi que sur la violence et les émeutes qui s'ensuivirent : «l'année affreuse », comme la qualifie un curé du Quercy: PAUMÈs, op. cit., p. 197. Voir LECLER, op. cit., p. 39 ; DUBOIS, op. cit., p. 51-52 ; MarieChristiane ARMOOGUM-NINAT, « La Grande Peur de 1789 à Sainte-Sévère (Indre) », A.H.R.F. 29 (1957), p. 122.

43. Voir, par exemple, Pierre VIDAL, Histoire de la Révolution française dans le département des Pyrénées-Orientales, 3 vol., Perpignan, 1885-1888, p. 60 ; Félix MOURLOT, La Fin de l'Ancien Régime et les débuts de la Révolution dans la généralité de Caen, Paris, 1913, p. 326-329 ; A.H.R.F. 10 (1933), p. 147-175.

44.DUBOIS, op. cit., p. 42 ; TRIGER, op. cit., p. 221. Voir les réactions similaires du Mans et de Villeneuve-sur-Yonne : TRIGER, op. cit., p. 223 ; et CHAUDRON, op. cit., p. 75. Voir aussi MÈGE, op. cit., p. 180, 183 ; LEFEBVRE, La Grande Peur, op. cit., p. 146 ; et SCHAMA, op. cit., p. 434. Le 
18 juillet, le député Duquesnoy écrit à son correspondant provincial : « Il n'y a plus de roi, plus de parlement, plus d'armée, plus de police... L'anarchie augmente, elle ira toujours en augmentant » : Adrien-Cyprien DUQUESNOY, Journal d'Adrien Duquesnoy, éd. R. de Crèvecoeur, 2 vols, Paris, 1894, vol. 1, pp. 231-232.

45.De fait, LEFEBVRE lui-même fournit probablement plus de preuves relatives à la peur de brigands expulsés ou quittant Paris qu'à des rumeurs d'un complot aristocratique en province : LEFEBVRE, La Grande Peur, pp. 148-155. Voir aussi, par exemple, CHAUDRON, op. cit., p. 33 ; PICKFORD, op. cit., « The Panic in Lower Dauphiné and in Provence », pp. 282-283 ; DINET, op. cit., La Grande Peur dans la généralité de Poitiers, p. 60 ; « Glanes [pour l'Eure-et-Loir] », A.H.R.F. (1935), p. 256. Pickford insiste tout particulièrement sur la peur du chaos et de l'anarchie dans son analyse des origines de la peur : voir en particulier « The Panic in Touraine », pp. 720-721.

46.Sarah MAZA, « Luxury, Morality and Social Change », JMH 69 (1997), p. 203 ; voir également Timothy TACKETT, Par la volonté du peuple, op. cit., pp. 100-104.

47.MÉNARD, p. 161 (lettre du 22 déc. 1789) ; voir aussi p. 38 et 95 (lettres du 5 juin et du 1er sept. 1789).

\section{RÉSUMÉS}

L'article discute la thèse célèbre de Georges Lefebvre sur le rôle d'un "complot aristocratique" dans les origines de la Grande Peur. En fait, en dehors de Paris, les preuves documentaires d'une croyance à un tel complot avant le déclenchement de la panique semblent être assez maigres. On trouve, en revanche, au beau milieu de la Peur, beaucoup d'exemples de solidarité verticale entre noblesse et roturiers. Les soupçons de complots sont en fait souvent apparus, par la suite, au fur et à mesure que les gens essayaient d'expliquer ce qui venait de se passer. La "cause" la plus vraisemblable de la Grande Peur est l'inquiétude, très répandue au début de la Révolution, face à l'instabilité, à l'anarchie, au vide du pouvoir, une inquiétude nettement accentuée par la crise de juillet. Dans beaucoup de provinces, l'obsession d'un complot aristocratique ne serait ainsi apparue que plus tard, dans les mois et années qui suivent l'été 1789.

The Great Fear and the Aristocratic Plot in the French Revolution. This article discusses Georges Lefebvre's celebrated thesis on the role of the fear of an «aristocratic plot» in the origins of the Great Fear. In fact, with the exception of Paris, the empirical evidence for a belief in such a plot before the outbreak of the panic is surprisingly thin. Rather the evidence commonly reveals a vertical solidarity between aristocracy and commoners in the very midst of the Fear. If theories of an aristocratic plot did arise, they generally appeared after the fact, as people attempted to explain what had happened. Ultimately, the most likely «cause» of the panic was the pervasive fear of instability, anarchy, and a power vacuum, enormously accentuated by the mid-July crisis. In many provinces, an obsession with aristocratic plots probably appeared only later, during the months and years that followed the summer of 1789. 
INDEX

Mots-clés : Grande Peur, panique, complot, Georges Lefebvre, insurrection, aristocratie, anarchie 